\title{
Dual Safeguard: Intrusion Detection and Prevention System in Web Applications
}

\author{
M.Sujitha \\ PG Scholar \\ Shivani Engg College \\ Trichy, Tamilnadu
}

\author{
P.Suganya \\ PG Assistant \\ Shivani Engg College \\ Trichy, Tamilnadu
}

\author{
T.Shampavi \\ PG Scholar \\ Shivani Engg College \\ Trichy, Tamilnadu
}

\author{
S.Anjanaa \\ PG Scholar \\ Shivani Engg College \\ Trichy, Tamilnadu
}

\begin{abstract}
Web applications are the most universal way to make services and data accessible on the Internet. With the increase in the use of these applications, there has also been an increase in the amount and complexity of vulnerabilities and attacks. These attacks target directly the back-end database systems of the web application to achieve information. In this paper, a Dual Safeguard Intrusion Detection and Prevention System was proposed to models the user network behaviors and create normality models of isolated user sessions across both frontend and back-end of the application. To accomplish this, a Lightweight virtualization technique is used to allocate each user's web session to a dedicated container, providing an isolated virtual computing environment. An Intrusion Detection Model for web applications based on Hidden Markov Model was also proposed.
\end{abstract}

\section{General Terms}

Attacks, Container, Intrusion Prevention, User's web session, Virtualization, Vulnerabilities.

\section{Keywords}

IDS, Multitier web application, Normality model, Hidden Markov Model, Container Virtualization.

\section{INTRODUCTION}

An essential part of our day-to-day life is Information Technology. There are a number of Online Transaction sites, Social Networking sites and other web portals were developed. Large amounts of data are stored in the databases of these web portals [6]-[8], the working of this portals are based around web technologies such as PHP, ASP.NET, JSP, XHTML and SQL. Security of a web application should be focused at the time of progress of the application itself. Since most of the web application is largely open, it is very easy to find security loopholes and turned as insecure web applications into potential victims for exploitation using worm attacks, etc.

To protect multitier web services, Intrusion detection systems have been broadly used to detect known attacks by matching misused traffic patterns or signatures. An IDS consists of a set of tools that can be used to detect and prevent attempts of intrusion. IDS can be distinguished based on anomaly-based and misuse-based Systems or signature based systems. The anomaly-based approach has been the initial system to be developed. Anomalous behaviors were classically related to intrusions, but they may also be associated to normal activities because the definition of a good model of normal activities is far away from being ideal.

Anomaly based systems could produce a high percentage of false alarms. For this reason, the most broadly used IDS model are based on misuse detection system. Misuse-based systems performs pattern matching among a set of rules (called signatures), which describes as well known attacks, and currently observed patterns. It is easy to see that misuse based IDS can exactly detect the known intrusions, but if the behavior of attacks are somewhat modified then the matching process is possibly to be unsuccessful. The increasing count of these kind of attacks in recent years motivates a rehabilitated interest on anomaly based IDS.

Individually, the web IDS and the database IDS can be able to detect abnormal network traffic sent to either of them. But these Intrusion Detection Systems cannot detect the cases in which normal traffic is used to attack the webserver and the database server. Unfortunately, within the present multithreaded webserver architecture, it is not feasible to detect or contour such causal mapping between webserver traffic and DB server traffic because traffic cannot be clearly recognized to user sessions.

In this paper, Dual Safeguard is presented, a system used to detect attacks in multitier web services. This approach can produce normality models of isolated user sessions that include both the web front-end (HTTP) and back-end (File or SQL) network transactions. To attain this, a light-weight virtualization technique was employed to assign each user's web session to a dedicated container, providing an isolated virtual computing environment. Thus, Dual Safeguard can build a causal mapping profile by taking both the web server and DB traffic into report. An anomaly based IDS was also proposed to analyze sequences of commands swapped between two hosts through a certain protocol (e.g., FTP, SMTP, HTTP, etc.), and produces an output score that is used to evaluate if the analyzed sequences are normal or anomalous. To model normal network traffic, Hidden Markov Models (HMM) was used. After a sequence of commands are analyzed, the HMM assigns a probability value that can be interpreted as the possibility that the sequence is normal. By setting a threshold on this probability value, it is possible to 
find out anomalous traffic. The container-based web architecture provides the profiling of causal mapping. Within the Container virtualization environment, many copies of the web server instances are executed in different containers so that each instance was isolated from other instances. As shortlived containers can be easily instantiated and destroyed, each client session was assigned a dedicated container, for the reason that when an attacker tries to compromise a single session, the damage is restricted to that compromised session; other user sessions remain unaltered by the attacks.

\section{LITERATURE SURVEY}

Executing malicious statements on a database may result in severe problems, which can range from exposure of sensitive information to losing records or broken integrity [4]. Once an attacker manages to inject code into a database this will likely not only affect specific records, but may lead to a compromise of the complete application environment. This in turn can cause stern damages to data records and company's public status. Although the risk may seem low on a first glance, given the database layer is separated from the public interface and not directly accessible from the external, abnormal queries caused by e.g. SQL injection attacks [11] are a widespread problem.

Anomaly detection techniques are prone to both false positives and false negatives. As anomaly based detection such as in [5], the proposed system composed of web-based IDS, a reverse HTTP proxy, and a database IDS. Its Webbased anomaly detector and a SQL query anomaly detector increase the detection rate of this system. The system allows to route anomalous requests to servers that have limited access to sensitive information. But its constraint shows that there is no mapping model between the Web-based anomaly detector and the Query anomaly detector to identify attacks.

The problem of mining frequent damage spreading patterns from the previous attack histories is given at [2]. A database firewall framework is devised to dynamically enforce a policy of transaction filtering to filter out the potential spreading transactions. This considers IDS for the back-end SQL queries alone and identifies only the patterns that cause damages to the data. Its performance is not good on heavy attacks and the computation overhead could increase when discovered spreading patterns accumulate.

Virtualization technique [3] is used to isolate objects and enhance security concert. Full virtualization and paravirtualization are the initial approaches being considered. An alternative now used is a Lightweight virtualization, such as OpenVZ [10], Parallels Virtuozzo, or Linux-VServer. With containers, a collection of processes still appears to have its own dedicated system, but it is running in an isolated environment. HMM [12] have also been used for Intrusion Detection due to their ability to model time-series using a stateful approach where the role and meaning of the internal states are "hidden". Previously, Snort IDS [13] is used to protect front end web server.
CLAMP [14] is architecture for preventing data leaks even in the presence of attacks. CLAMP requires alteration to the existing application code, and the Query Restrictor works as a proxy to decide all database access requests. This approach uses process isolation whereas CLAMP involves platform virtualization, and provides more coarse-grained isolation than Dual Safeguard.

Green SQL [9], works as a reverse proxy for database connections. Instead of connecting to a database server, web applications will directly connect to a database firewall. SQL queries are analyzed; if they are found safe, then the queries are forwarded to the back-end database server.

\section{SYSTEM ARCHITECTURE}

Both the web and the database servers are vulnerable to attacks. The attackers can evade the web server to directly attack the database server. The attacks can neither be detected nor be prevented by the current web server IDS, those attackers may take over the web server after the attack.

\subsection{Architecture}

In this system, lightweight process called 'Containers', as short-lived, disposable servers for client sessions was used. A single physical web server runs many containers; each container has an exact copy of the original web server. This approach dynamically creates new containers and recycles the used containers. Sessions can represent different users and the communication of a single user likely to go to the same dedicated web server, in this manner allowing us to identify expect behavior by both session and user. If abnormal behaviors in a session were noticed, treat all traffic within this session as contaminated. An attacker can only stay within the web server container that is connected. Thus ensure that valid sessions will not be compromised by an attacker directly.

Fig. 1 shows how communications are categorized as sessions and how the database transactions can be related to a corresponding session. Here client 2 will compromise the VE 2 , and the corresponding database transaction set $\mathrm{T} 2$ will be the only affected section of data within the database.

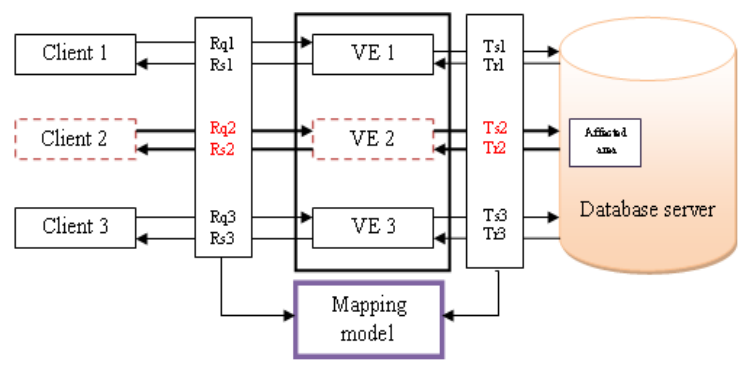

Fig 1: Container based architecture

\subsection{Attacks over the web}

This system is effectual at capturing the following types of attacks: 


\subsubsection{Privilege Escalation Attack}

For a normal user, the web request $r_{u}$ will prompt the set of SQL queries $Q_{u}$; for an administrator, the request $r_{a}$ will activate the set of admin level queries $\mathrm{Q}_{\mathrm{a}}$. Assume that an attacker logs into the web server as a normal user, improves their privileges, and triggers admin queries as a result to obtain an administrator's data. This attack cannot be detected by either the web server IDS or the database IDS because both $\mathrm{r}_{\mathrm{u}}$ and $\mathrm{Q}_{\mathrm{a}}$ are authentic requests and queries. This approach, can detect this type of attack in view of the fact that the DB query $Q_{a}$ does not match the request $r_{u}$, according to the mapping pattern.

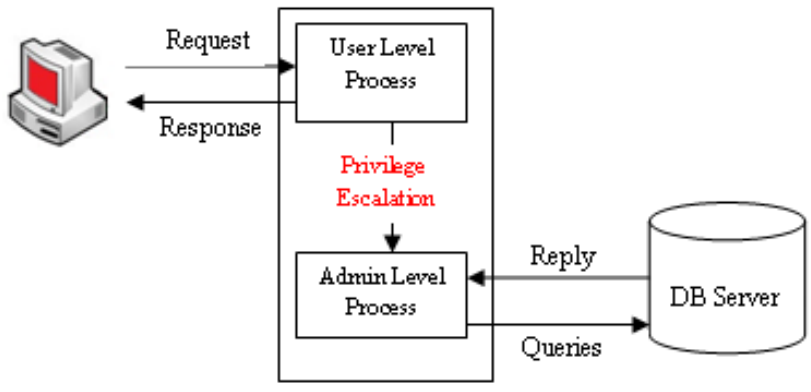

Fig 2: Privilege Escalation Attack

\subsubsection{Injection Attack}

Attackers can use accessible vulnerabilities in the web server logic to infuse the data or string that contains the abuse and then use the web server to relay these exploits to attack the back-end database. Since the SQL injection attack modifies the SQL queries structure, it would generate SQL queries in a structure that could be noticed as a deviation from the SQL query structure.

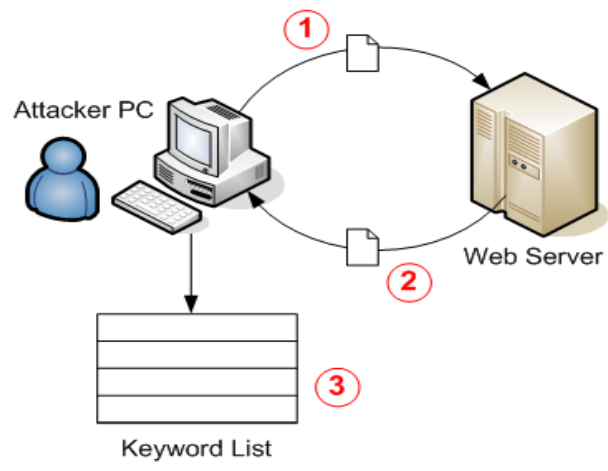

Fig 3: Injection Attack

\subsubsection{Hijack Future Session Attack}

This class of attacks is mainly aimed at the web server side. An attacker will usually grab the web server and then hijacks all subsequent valid user sessions to initiate attacks. For example, by capturing other user sessions, the attacker can snoop, send spoofed replies, and/or drop user requests. A session-hijacking attack can be further categorized as a Spoofing/Man-in-the-Middle attack, a Denial-of-Service or a Replay attack or a Packet Drop attack.

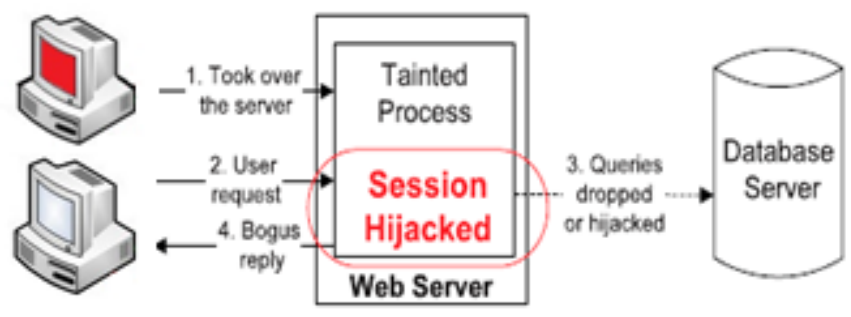

Fig 4: Hijack Future Session Attack

\subsubsection{Direct DB Attack}

It is possible for an attacker to bypass the web server or firewalls and connect directly to the database. An attacker takes over the web server and submitting queries from the web server without sending web requests.

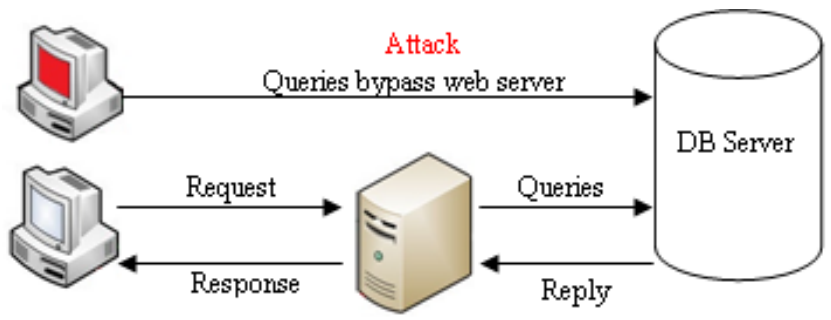

Fig 5: Direct DB Attack

\subsubsection{DDOS Attack}

Distributed Denial of Service, is a type of DOS attack where multiple compromised systems -- which are frequently infected with a Trojan. Trojans are used to target a single system causing a Denial of Service (DoS) attack. Fatalities of a DDoS attack consist of both the end targeted system and all systems maliciously used and controlled by the hacker in this distributed attack.

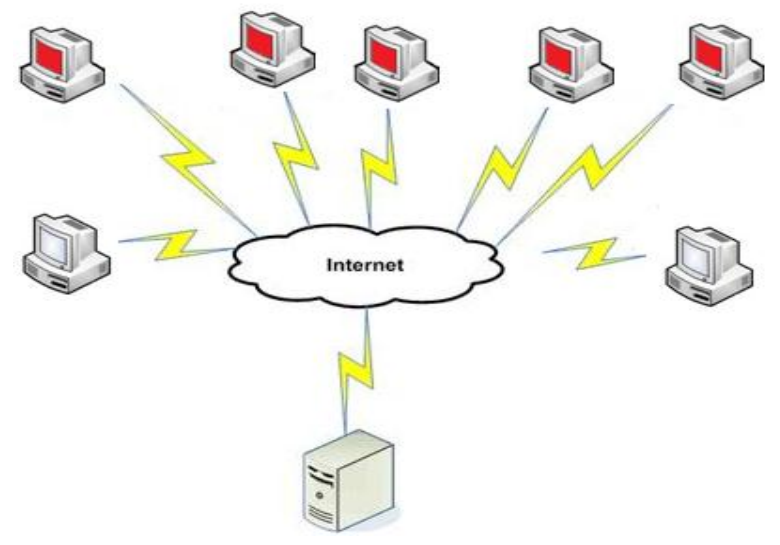

Fig 6: DDOS Attack

\section{MAPPING MODELS}

For every session $i$ have a set of requests $\mathrm{R}_{\mathrm{i}}$, as well as a set of queries $\mathrm{Q}_{\mathrm{i}}$. If the total number of sessions of the training phase is $\mathrm{N}$, then the set of total web requests REQ and the set of total SQL queries SQL present at all sessions. Each single 
web request $r_{m} \in$ REQ may also appear several times in different Ri where i can be $1,2 \ldots$. The same holds true for $\mathrm{q}_{\mathrm{n}} \in \mathrm{SQL}$. The mappings in the model are always in the form of one request to a query set $r_{m} \rightarrow Q_{n}$. The possible mapping patterns are as follows:

\subsection{Deterministic Mapping}

It is the most commonly used and perfectly matched pattern. The mapping pattern is then $r_{m} \rightarrow Q_{n}\left(Q_{n} \neq 0\right)$. Mostly used in static websites since the same results should be returned for each time a user visits the same link.

\subsection{Empty Query Set}

Here the SQL query set may be the empty set. Web request neither causes nor generates any database queries. The mapping pattern is then $r_{m} \rightarrow \emptyset$. These web requests are kept together in the set EQS.

\subsection{No Matched Request}

This mapping is not driven by any web request. It is similar to the reverse case of the empty query set mapping model. These queries cannot coincide with any web requests. These unmatched queries are kept in a set NMR.

\subsection{Nondeterministic Mapping}

The same web request may result in different SQL query sets based on input parameters or the status of the webpage at the time the web request is received. The mapping pattern is then $\mathrm{r}_{\mathrm{m}} \rightarrow \mathrm{Q}_{\mathrm{i}}\left(\mathrm{Q}_{\mathrm{i}} \in\left\{\mathrm{Q}_{\mathrm{n}}, \mathrm{Q}_{\mathrm{p}}, \mathrm{Q}_{\mathrm{q}} \ldots \ldots\right\}\right)$. This happens only within dynamic websites.

\subsection{Hidden Semi-Markov Model}

HsMM is an extension of the Hidden Markov Model with clear state duration. It is a stochastic finite state machine, specified by (S, П, A, P) where:

- $\quad \mathrm{S}$ is a discrete set of hidden states with cardinality $N$, i.e., $S=\{1, \ldots ., N\}$;

- $\Pi$ is the probability distribution for the initial state;

- A is the state transition matrix;

- $\quad \mathrm{P}$ is the state duration matrix.

\section{IMPLEMENTATION OF PROPOSED METHODOLOGY}

In This implementation, containers were recycled based on events or when sessions time out. The same session tracking mechanisms as implemented by the Apache server can be used because lightweight virtualization containers do not impose high memory and storage overhead.

\subsection{Modeling and Testing for Static Websites}

For each $r_{m} \in R E Q$, A set ARm was maintained to record the IDs of sessions in which $r_{m}$ appears. The same holds for the database queries; a set AQs for each $\mathrm{q}_{\mathrm{s}} \in \mathrm{SQL}$ to record all the session IDs. For each ARm, the AQs that equal the Arm was searched. When ARm $=A Q s$, this indicates that every time $r_{m}$ appears in a session, then $\mathrm{q}_{\mathrm{s}}$ will also appear in the same session, and vice versa. For each unique HTTP request and database query, the algorithm allocates a hash table entry, the key of the entry is the request or query itself, and the value of the hash entry is $\mathrm{AR}$ for the request or $\mathrm{AQ}$ for the query, respectively.

\section{Static Model Building Algorithm}

a. Collect each session's requests and the queries.

b. For each request in each session do

- Check whether the request $r$ is for static file

- If so, add it to Empty Query set

- Else, if $r$ is not a request

- Then add to REQ, and append container with id I with $r$ as key.

c. For each Query in each session do

- $\quad$ Check whether the query q is not present.

- If so, add it to SQL

- Then append it to the container with id I with $\mathrm{q}$ as key.

d. For each request select one query

e. Compare request with the query

f. Check for deterministic mapping and add to the mapping model.

g. If no deterministic mapping is found then add to the empty query set.

During the testing phase, each session is compared to the normality model. The mapping model contained the Deterministic Mappings and Empty Query Set patterns without the No Matched Request pattern. Any untested web request or unmarked database query is considered to be anomalous. If any survives within a session, then that session will be marked as wary.

\subsection{Modeling and Testing of dynamic websites}

In contrast to static WebPages, dynamic WebPages allow users to generate the same web query with different parameters. Dynamic pages often use POST rather than GET methods to commit user inputs. Our blog website built for testing purposes shows modeling nine basic operations, can cover most of the operations that appeared in the real captured traffic. Notice that this mapping model includes both deterministic and nondeterministic mappings and the set EQS is still used to hold static file requests. Dynamic model may incur false positives. For the testing session i, the set of DB queries Qi should be a subset of the CQS (Entire Corresponding Query Sets). For the web requests in Ri, each should either match at least one request in the operation model or be in the set EQS. If any unmatched web request leftovers then the session has violated the mapping model.

\subsection{Identifying Hidden Attacks}

This model aims at detecting intrusions by analyzing the sequences of commands that flow between the hosts in a network for a particular service. The system must be trained in order to learn the typical sequences of commands related to inoffensive connections. The component Data-Preprocessing, 
performs a number of preliminary operations on the sequence in order to make it suitable for the HMM.

During the training phase, this module extracts the dictionary of symbols to be used by the HMM. HMM are built using a set of training sequences. Once the model is built, its performances are assessed by a set of test sequences. For every test sequence, the HMM outputs a probability value stating that how likely the sequence is anomalous. By setting a decision threshold value, the sequence can be found out as normal or anomalous session. If it found anomalous then that session will contain hidden attacks.

\section{PERFORMANCE EVALUATION}

A prototype of Dual Safeguard was implemented using a webserver with a back-end DB. Two testing websites, one static and the other dynamic was arranged. For occasion, assign a new container per each new IP address of the client. Here containers were recycled based on events or when sessions time out.

One of the main concerns for a security scheme is its performance overhead in terms of latency. In this case, even though the containers can start within seconds, generating a container on the fly to serve a new session will increase the response time profoundly. To ease this, a pool of webserver containers was created for the forthcoming sessions. The overhead of the container-based server was evaluated against a usual webserver. In order to measure throughput and response time, http_load benchmark tool was used.

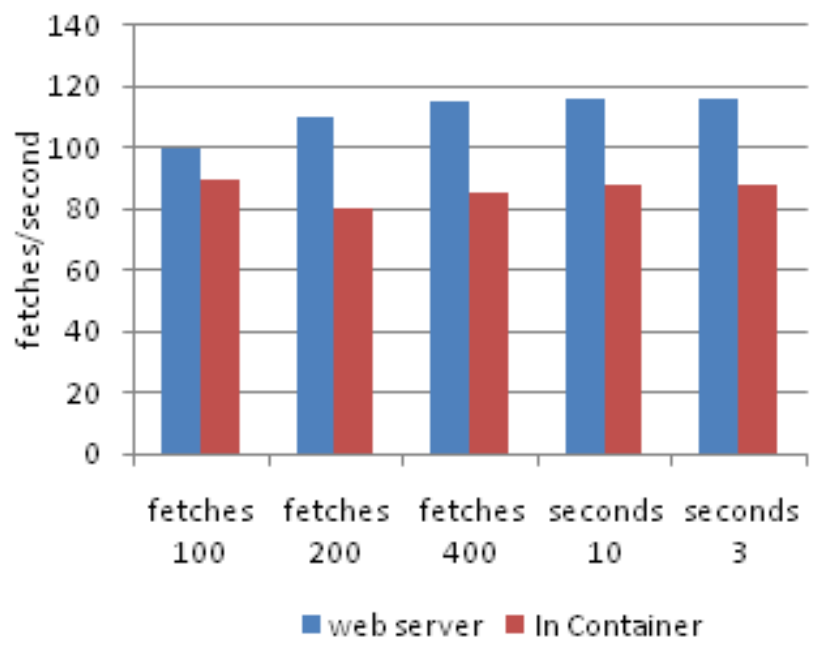

Fig 7: Performance Evaluation using http_hoad

The value of fetches per second in the http_load results is the most essential indicator to reflect webserver throughput performance.

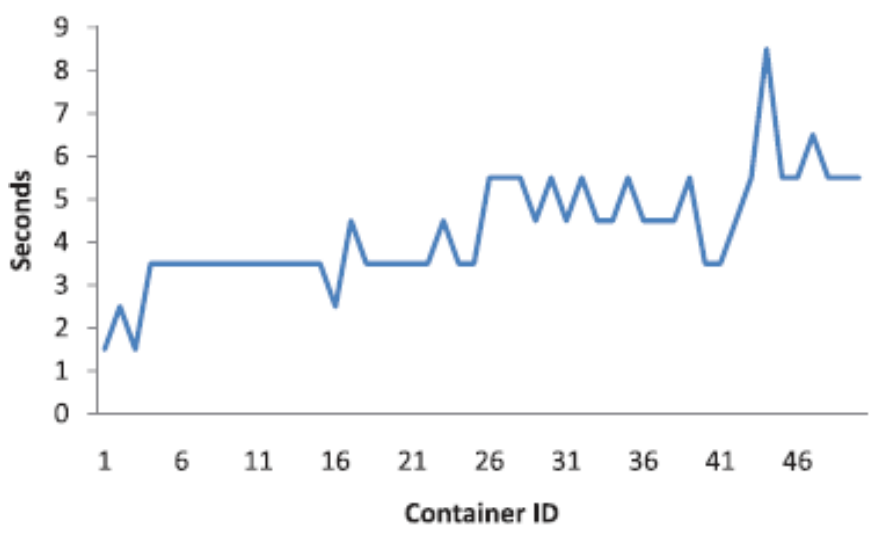

Fig 8: Time to start a new container

Fig 8 shows the time needed for starting a container. Since 50 containers were opened in a row, the average time was about 4.2 seconds. Thus the false positive rate is reduced much and the efficiency of detecting more number of attacks was improved efficiently.

\section{CONCLUSION}

IDS that provides models of normal behavior of web applications obtained from both front-end web (HTTP) requests and back-end database (SQL) queries was presented. Dual Safeguard forms container-based IDS with multiple input streams. However, such relationship of inputs gives a enhanced characterization of the system for anomaly detection. The more specific normality model detects a wider range of threats and is achieved by isolating the flow of information from each web server session with a Container virtualization technique.

In addition, the detection accuracy of this approach is also enumerated when attempted to model static and dynamic web requests with the back-end file system and database queries. For static websites, a well-correlated model is built, to be efficient at detecting different types of attacks. Additionally, this held true for dynamic requests where both retrieval of information and updates to the back-end database done using the web server front end. Dual Safeguard was able to identify a wide range of attacks with minimal false positives. To avoid hidden attacks which are not easily identified are tried to found using Hidden Semi Markov Model. Finally, for dynamic web applications, the false positives are reduces from 0.6 percent to minimum.

\section{REFERENCES}

[1] M. Le, A. Stavrou and B. B. Kang, "DoubleGuard: Detecting Intrusions in Multitier Web Applications", IEEE Transactions On Dependable And Secure Computing, vol. 9, no. 4, July/August 2012

[2] K. Bai, H. Wang, and P. Liu, "Towards Database Firewalls," Proc. Ann. IFIP WG 11.3 Working Conf. Data and Applications Security (DBSec '05), 2005.

[3] Y. Huang, A. Stavrou, A.K. Ghosh, and S. Jajodia, "Efficiently Tracking Application Interactions Using 
Lightweight Virtualization," Proc. First ACM Workshop Virtual Machine Security, 2008.

[4] R. Sekar, "An Efficient Black-Box Technique for Defeating Web Application Attacks," Proc. Network and Distributed System Security Symp. (NDSS), 2009.

[5] G. Vigna, F. Valeur, D. Balzarotti, W.K. Robertson, C. Kruegel, and E. Kirda, "Reducing Errors in the Anomaly-Based Detection of Web-Based Attacks through the Combined Analysis of Web Requests and SQL Queries," J. Computer Security, vol. 17, no. 3, pp. 305-329, 2009.

[6] SANS, "The Top Cyber Security Risks," http://www.sans.org/top-cyber-security-risks/, 2011.

[7] "Common Vulnerabilities and Exposures," http://www.cve.mitre. org/, 2011.
[8] "Five Common Web Application Vulnerabilities," http://www.symantec.com /connect/articles/fivecommon-web application vulnerabilities, 2011.

[9] Greensql, http://www.greensql.net/, 2011.

[10] Openvz, http://wiki.openvz.org, 2011.

[11] A. Schulman, "Top 10 Database Attacks," http://www.bcs.org/server.php, 2011.

[12] W. Robertson, F. Maggi, C. Kruegel, and G. Vigna, "Effective Anomaly Detection with Scarce Training Data," Proc. Network and Distributed System Security Symp. (NDSS), 2010.

[13] M. Roesch, "Snort, Intrusion Detection System," http://www.snort.org, 2011.

[14] B. Parno, J.M. McCune, D. Wendlandt, and A. Perrig, "CLAMP: Practical Prevention of Large-Scale Data Leaks," Proc. IEEE Symp. Security and Privacy, 2009. 